1. FCPS, FCPS

Assistant Professor Cardiac

Surgery

Choudhry Pervaiz Elahi Institute of Cardiology, Multan

2. FCPS, FCPS

Senior Registrar Cardiac Surgery Choudhry Pervaiz Elahi Institute of Cardiology, Multan

3. Medical Officer Cardiac Surgery Choudhry Pervaiz Elahi Institute of Cardiology, Multan

Correspondence Address: Dr. Naseem Ahmad

Assistant Professor Cardiac Surgery Choudhry Pervaiz Elahi Institute of Cardiology, Multan

drnaseemahmad@yahoo.com

\section{MITRAL VALVE REPLACEMENT ON BEATING HEART; SAFETY-------AN EVALUATION}

\section{Dr. Naseem Ahmad', Dr. Ghulam Hussain ${ }^{2}$, Dr. Muhammad Naveed Shahzad ${ }^{3}$}

ABSTRACT: Introduction: Cardiac reperfusion injury is a well-described complication occurring after ischemia or following cardioplegic arrest. The primary aim of this study was to evaluate and compare safety of Mitral valve replacement on beating heart without using cross clamp with the conventional Mitral valve replacement. Material and methods: It was a retrospective observational study for study duration from 2012-2014. A total of 50 patients were randomly selected and were divided in 2 groups, 25 patients in each group. This study was conducted at the Department of Cardiovascular Surgery of Choudhry Pervaiz Elahi Institute of Cardiology, Multan. We divided the patients in two groups. All operations were carried out by team led by a consultant surgeon in our institution. Results: Our patients had the age range from 12 to 65 years (Mean ages $=32.5$ years \pm 13.9000 ) the mostly this study included males $60 \%$. The study included Group A and Group B (beating heart versus conventional heart surgery) both groups contained randomized controlled patients with purposeful sampling. Minimum operative time 100 minutes or maximum operative time 160 minutes (Mean 195 195.75 ) The $P$ value remained non significant that $P<0.001$. The CPB time was 22 to 388 minutes (104.8 \pm 97.4 . Cross Clamp time, ICU stay intubation time, drainage, inotrope remained almost same in both groups. With $P$ $<0.001$ Outcome variables of the patients. Discussion: The hazards of cardiopulmonary bypass and Cardioplegia are well known. Various studies have demonstrated decreased accumulation of extra cellular fluid, diminished lactate production and greater preservation of high energy stores when a strategy of myocardial protection simultaneous antegrade/ retrograde continous normothermic, normokalemic blood perfusion was used. These findings were the basis for using beating heart technique for mitral valve replacement, Aortic cross-clamping, Cardioplegia, and reperfusion injury leads to myocardial ischemia and is a critical issue in mitral valve surgery despite novel approaches to myocardial protection. Myocardial edema induced by the lack of myocardial contractions and impaired lymphatic flow due to Cardioplegia in the heart remaining in diastole is another cause of myocardial dysfunction. Conclusions: Outcomes obtained using this strategy of myocardial protection seem to compare favorably to those of historical series in which conventional myocardial protection with cardioplegic arrest were used.

Key words: $\quad$ Mitral Valve Replacement, Cardio-Pulmonary Bypass (CPB)

Article Citation: Ahmad N, Hussain G, Shahzad MN. Mitral valve replacement on beating heart; safety ------- an evaluation. Professional Med J 2014;21 (5):1015-1020.

During beating-heart valve surgery, the heart is kept empty and allowed to beat continuously, while myocardial protection is achieved through continuous coronary perfusion of the heart, as opposed to operating on a still heart in a state of cardioplegic arrest. Prior to the introduction of Cardioplegia, beating heart continuous coronary perfusion was the only available method of myocardial protection. The interest in beating heart continuous coronary perfusion as an alternative to Cardioplegia in valvular surgery stems from the increased familiarity with the off pump technique, an improved understanding of the potential harms of Cardioplegia (e.g. 
retrograde perfusion injury) and the development of improved perfusion technique (e.g. retrograde perfusion $)^{3}$. This technique of beating heart has been observed to decrease the risk of uncontrolled myocardial ischemia, particularly in patients with borderline myocardial function and provides better myocardial protection and early improvement. In addition to avoiding reperfusion injury, on pump beating heart valvular operations may have other advantages because of the fact that the heart is under more physiologic conditions then the cardioplegic arrested state with left ventricular beating tonus. The primary aim of this study was to evaluate and compare safety of Mitral valve replacement on beating heart without using cross clamp with the convention Mitral valve replacement in terms of post operative Neuro Cognitive function, ICU stay, ventilation time, inotropes time and CPK and CKMB levels. (Simply cardiac enzymes should be operated defunctions ${ }^{1-8}$.

\section{MATERIAL AND METHODS}

It was a retrospective observational study for study duration from 2012-2014. A total of 50 patients were randomly selected and were divided in 2 groups, 25 patients in each group. This study was conducted at the Department of Cardiovascular Surgery of Choudhry Pervaiz Elahi Institute of Cardiology, Multan. Our inclusion criteria was The patients who underwent Mitral valve replacement (MVR) for pure Mitral regurgitation of any age and sex.

Exclusion Criteria were, All diabetic patients, Redo MVR,E.F $<30 \%$,Patient with stenotic and calcified valves, Patients having tricuspid regurgitation, Patients with involvement of aortic valve, Patients having severe pulmonary hypertension, Who underwent emergency or salvageable surgery?

We divided the patients in two groups.

- Group A: The patients who underwent beatingheart mitral valve replacement without cross clamping the aorta.

- Group B: The patients who underwent mitral valve replacement procedure via the standard ascending aorta-cannulation technique. (On

\section{pump surgery)}

The safety and efficacy of mitral valve replacement on beating heart without using cross clamp as assessed in terms of post operative neurocognitive function, ICU stay, ventilation time, inotropes time and $\mathrm{CPK}$ and CK-Mb levels and was compared with the other group of patients.

All operations were carried out by team led by a consultant surgeon in our institution. Patients were premeditated with Lexilium $3 \mathrm{mg}$ orally the night before surgery. Anesthesia was induced with morphine $0.1 \mathrm{mg} / \mathrm{kg}$, midazolam $0.05-0 . \mathrm{mg} /$ $\mathrm{kg}$, propofol $1.0-2.5 \mathrm{mg} / \mathrm{kg}$ (as titration dose) and atracuronium $1 \mathrm{mg} / \mathrm{kg}$ (intubating dose) and was maintained with sevoflurane or isoflurane. Further supplementation of analgesia and paralyzing agent were given as needed.

The standard cardiopulmonary bypass (CPB) was established with aortic and 2 stage right atrial cannula. The CPB circuit was primed with crystalloid ringer's solution. The Heparin was administered in a dose of $400 \mathrm{U} / \mathrm{kg}$. The systemic temperature was not lowered and the operation was conducted on normothermia. All the patients underwent elective mechanical ventilation until they were ready for extubation after surgery in ICU. The patients were placed in the Trendelenburg position to prevent air embolism. We also used aortic and pulmonary vents for removal of air, maintained until the patients were weaned from CPB. The ascending aorta was not clamped and Cardioplegia was not used; consequently, the heart was not fibrillated. A left atriotomy was performed and a vent was placed in the left atrium (LA) via the right upper pulmonary vein and the aortic vent was also used. The left heart was kept empty and vented to the atmosphere to avoid air embolism. With this approach, the blood flows through the mitral valve where the atmospheric pressure is lower and cannot pass through the aortic root since the atmospheric pressure is higher.

In patients placed in group $B$, the standard cardiopulmonary bypass (CPB) was established 
with aortic and 2 stage right atrial cannulae. The CPB circuit was primed with crystalloid ringer's solution. The Heparin was administered in a dose of $400 \mathrm{U} / \mathrm{kg}$. The systemic temperature was lowered to 30 to $32 \mathrm{C}$. The local cooling was done with ice slush. CPB was initiated and aortic cross clamp was placed. Tepid blood cardioplegia was given through cardioplegia cannula in the ascending aorta and was repeated every 20 minutes.1st dose of cardioplegia was $10-15 \mathrm{ml} /$ $\mathrm{kg}$ and next repeated dose was $5-7 \mathrm{ml} / \mathrm{kg}$. All the patients underwent elective mechanical ventilation until they were ready for extubation after surgery in ICU.

During ICU stay all patients had invasive and noninvasive hemodynamic monitoring. When they were extubated and hemodynamically stable, they were shifted to high dependency unit named as ICU II in our institution. We also recorded the age, weight, height, aortic cross clamp time, bypass time, any arrhythmias after aortic unclamping, inotropic and mechanical support needed at time of weaning from bypass and duration of support. We recorded the total drainage, the number of blood and the blood products used post operatively, the neurological status and maximum $\mathrm{CK} \mathrm{MB}$ in 24 hours. The data were entered in a Micro Excel sheet version 2007 prospectively and the statistical analysis was carried out using
SPSS version 15 . The continuous variables were compared by ANOVA. The analysis of variance with repeated measures was used to compare the trends in rise and fall of CPK and CP-MB levels.

The preoperative, operative and postoperative variables were summarized using means and standard deviation for the numeric variables. The groups were compared using Student's t-test for numeric variables. The significance of difference between the groups was expressed as p-value and a value of $<0.001$ was considered significant.

\section{RESULTS}

Our patients had the age range from 12 to 65 years (Mean ages $=32.5$ years \pm 13.9000 ) the mostly this study included males $60 \%$. The study included Group A and Group B (beating heart verses conventional heart surgery) both groups contained randomized controlled patients with purposeful sampling.

Minimum operative time 100 minutes or maximum operative time 160 minutes (Mean 195 \pm 95.75 ) The $P$ value remained non significant that $P<0.001$. The CPB time was 22 to 388 minutes (104.8 \pm 97.4. Cross Clamp time, ICU stay intubation time, drainage, inotrope remained almost same in both groups. With $\mathrm{P}<0.001$.

Outcome variables of the patients.

\begin{tabular}{|l|c|c|c|}
\hline Variables & Beating heart group & Conventional group & p-value \\
\hline Operative time (min) [median (min-max)] & $130(100-270)$ & $240(100-360)$ & $<0.001$ \\
\hline CPB time (min) [median (min-max)] & $57(22-150)$ & $90(39-388)$ & $<0.001$ \\
\hline Cross Clamp time (min) [median (min-max)] & 0 & $60.5(30-163)$ & $<0.001$ \\
\hline Length of ICU stay (day) (min) [median (min-max)] & $1(1-34)$ & $1(1-90)$ & 0.379 \\
\hline Intubation time (h) (min) [median (min-max)] & $(4-600)$ & $12(2-1440)$ & 0.005 \\
\hline Drainage volume (ml) (min) [median (min-max)] & $475(150-4500)$ & $550(100-5825)$ & 0.45 \\
\hline Transfusion volume (units) (min) [median (min-max)] & $3(1-12)$ & $3(1-32)$ & 0.045 \\
\hline Need for support n (\%) & & & $<0.001$ \\
\hline Inotropic & $20(16.0 \%)$ & $68(35.0 \%)$ & 0.957 \\
\hline Re-exploration n (\%) & $4(3.2 \%)$ & $6(3.1 \%)$ & 0.584 \\
\hline Major cerebrovascular event n (\%) & $3(2.4 \%)$ & $3(1.6 \%)$ & 0.584 \\
\hline Pulmonary complications n (\%) & $3(2.4 \%)$ & $3(1.6 \%)$ & \\
\hline
\end{tabular}




\section{DISCUSSION}

The hazards of cardiopulmonary bypass and Cardioplegia are well known. Various studies have demonstrated decreased accumulation of extra cellular fluid, diminished lactate production and greater preservation of high energy stores when a strategy of myocardial protection simultaneous antegrade/ retrograde continous normothermic, normokalemic blood perfusion was used. These findings were the basis for using beating heart technique for mitral valve replacement. Aortic cross-clamping, Cardioplegia, and reperfusion injury leads to myocardial ischemia and is a critical issue in mitral valve surgery despite novel approaches to myocardial protection. Myocardial edema induced by the lack of myocardial contractions and impaired lymphatic flow due to Cardioplegia in the heart remaining in diastole is another cause of myocardial dysfunction ${ }^{9-14}$.

Reperfusion injury is inevitable when aortic crossclamp is removed in the arrested heart. Keeping the heart beating results in less myocardial edema and better cardiac function ${ }^{4}$.

Normothermic perfusion reduces the risks of induced hypothermia which includes allowing the myocardium to beat at normothermia with maintenance of normal coronary perfusion. It offers optimal myocardial protection with better preservation of myocardial blood flow distribution. This is off particular importance in patients with long-standing mitral valve disease who frequently have impaired ventricular function, a particular problem when such patients present in an emergency ${ }^{15-18}$.

In the present study, postoperative levels of creatine kinase $\mathrm{MB}$, troponin was significantly lower in the beating heart patients. Consistent with these results is the present finding that maintenance of the heart in a beating state throughout the operation results in less damage than cardioplegic arrest, even when blood Cardioplegia is used in a continuous fashion ${ }^{19-21}$.

The chief criticism in the literature regarding this approach is the risk for an air embolism. No single patient $(0 \%)$ in our 25 of 50 patients study group had major cerebrovascular events. It is evident from the literature that major cerebrovascular events after open heart surgery varies between I and $4 \%{ }^{5}$. Our study group's major cerebrovascular events ratio is correlated with the literature an also none of them was caused by air embolism that means major cerebrovascular events of the study group were unrelated with the technique used. Marco Ricci et al in a study of 59 patients operated on beating heart technique did not observe neurologic deficits in relation to air embolism, a potential concern when performing beating heart mitral valve surgery without clamping the aorta $^{6}$. Likewise in another study conducted by Thompson et al. on 125 patients by utilizing the same approach, major cerebrovascular events were noted in two patients only $(1.6 \%)^{7}$. These studies and our data support the fact that no major cardiovascular complications are associated with this technique $22-24$.

In our study, the bypass time was significantly lower in patients with beating mitral valve replacement. Likewise, they required inotropes for less amount of time post operatively as compared to the other group. Thompson et al also experienced a relatively low inotropes support after revisional mitral valve surgery $(28.8 \%)$ using beating heart technique.

Another possible contraindications to beating heart technique during mitral valve surgery is the inability to obtain adequate visualization of the mitral valve apparatus and the presence o extensive mitral valve vegetations. Because of our venting technique. We have not experienced problems concerning visualization of the surgical site.

The beating mitral valve replacement yields low mortality and morbidity rates, it has shorter operative times, postoperative intubations time and length of hospital stay as compared to the conventional method. Furthermore, less drainage volume and less blood transfusion were observed in this group ${ }^{8}$. According to a study there are great advantages of beating heart valve surgery as for 
example, 1 the perfused myocardial muscle, 2 the heart is not doing any work, 3 no reperfusion, 4 the possibility for ablation of atrial fibrillation on the beating heart, and 5 testing of the mitral valve repair is done in real physiologic conditions in the state of left ventricle beating tonus. The procedure could be the procedure of choice for the valve operation or combined operation (valve operation and $\mathrm{CABG}$ ) in high-risk patients with low ejection fractions ${ }^{9}$. Kervan et al concludes that the technique of mitral valve replacement with normothermic CPB without cross-clamping of the aorta may be safely used for the majority of patients requiring mitral valve replacement without causing deterioration in neurocognitive function ${ }^{10}$. The advantages of on-pump beating mitral valve replacement are due to the fact that the heart is under more physiologic conditions than cardioplegic arrested state with left ventricular beating tonus, thus eliminating adverse effects of global myocardial ischemia produced from reperfusion injury. According to Kareem and David Taggart who presented a systematic review of the literature, beating-heart continuous coronary perfusion is an operative strategy in valve surgery with some potential benefits. There is, however a need for a high quality, prospective, randomized control trial to establish the exact role for BHCCP in complex valve surgery ${ }^{25-26}$.

\section{CONCLUSIONS}

Outcomes obtained using this strategy of myocardial protection seem to compare favorably to those of historical series in which conventional myocardial protection with cardioplegic arrest were used. Further studies are needed to fully evaluate the potential benefits of this method of myocardial perfusion as a means of eliminating ischemia-reperfusion injury in multiple valve surgery.

Copyright@ 28 July, 2014.

\section{REFERENCES}

1. Kaplon RJ, Pham SM, Salerno TA. Beating-heart valvular surgery: a possible alternative for patients with severely compromised ventricular function. J Card Surg 2002;17:170.

2. Katirciaglu SF, Cicekcioglu F, Tutun U, Parlar Al, Babaroglu S, Mungan U,Aksoyek A. On-pump beating heart mitral valve surgery without cross-clamping the aorta. J Card Surg. 2008 July-Aug;23(4):307-11.

3. Weman SM, Karhunen PJ, Penttila A, Jarvinen AA, Salminen US. Reperfusion injury associated with onefouth of deaths after coronary artery bypass grafting. Ann Thorac Surg 2000;70:807.

4. Mehlhorn U, Allen SJ, Adamus DL, Davis KL, Gogola GR, Warters RD. Cardiac surgical conditions induced by B-blockade: effect on myocardial fluid balance. Ann Thorac Surg 1996;62:143-50.

5. Halpey ZI Ganim RB, Rawn JD. Postoperative care of cardiac surgery patients. In: Cohn LH, editor. Cardiac surgery in the adult. New York: McGraw-Hill, 2008. p.465-86.

6. Multiple Valve Surgery with Beating Heart Technique. Marco Ricci, Francisco Igor B. Macedo, Maria R. Suarez, Micheal Brown, Julia Alba and Tomas A. Salerno Ann Thorac Surg 2007;87:527-31.

7. Thompson MJ, Behramwala A, Campanella C, Walker WS, Cameron FW. Immediate and long-term results of mitral prosthetic replacement using a right thoracotomy beating heart technique. Eur $\mathrm{J}$ Cardiothorac Surg 2003;24:47-51.

8. Babaroglua S, Yaya K, Parlara Al, Mungana CAU, Cicekcioglua F, Tutuna U, Katirciogluc SF. Beating heart versus convention mitral valve surgery. Interact Cardio Vasc Surg (2011);12(3)441-7.

9. Gersak B, Sutlic Z. Aortic and mitral valve surgery on the beating heart is lowering cardiopulmonary bypass an aortic cross clamp time. Heart Surg Forum. 200;5(2):182-6.

10. Kervan U, Cicekcioglu F, Tuluce H, Ozen A, Babaroglu S, Karakas S, Katircioglu SF. Comparison of neurocognitive function after beating-heart mitral valve replacement without aorta cross-clamping and after standard mitral valve replacement with cardioplegic arrest. Heart Surg Forum. 2011 Dec;14(6): E335-9.

11. Kareem Salhiyyah and David Taggart. Beating-heart Valve Surgery: A Systematic Review. Asian Cardiovase Thoracic Ann 2009;17:650.

12. Kaplon RJ, Pham SM, Salerno TA. Beating-heart valvular surgery: a possible alternative for patients with severely compromised ventricular function. J Card Surg2002;17:170-2.

13. Mehlhorn U, Allen SJ, Adams DL. Cardiac surgical conditions induced by beta-blockade: effect on myocardial fluid balance. Ann Thorac Surg 
1996;62:143-50.

14. Matsumoto $Y$, Watanabe G, Endo $M$ et al. Efficacy and safety of on-pump beating heart surgery for valvular disease. Ann Thorac Surg2002;74:678-83.

15. Eke CC, Gundry SR, Fukushima N, I. Is there a safe limit to coronary sinus pressure during retrograde cardioplegia? Am Surg 1997;63:417-20.

16. Tsukioka T, Tomita S, Watanabe $\mathrm{G}$ at al. Optimal continuous retrograde coronary perfusion flow rate with beating heart in normal temperature. Artif Organs2004;28:303-9.

17. Tian G, Xiang B, Dai G. The effects of retrograde cardioplegia technique on myocardial perfusion and energy metabolism: a magnetic resonance imaging and localized phosphorus 31 spectroscopy study in isolated pig hearts. J Thorac Cardiovasc Surg2000;120:544-51.

18. Tosson R, Kuschkowitz F, Dasbach G. Relationship between position of the coronary sinus catheter and distribution of cardioplegia. J Heart Valve Dis 1999;8:120-23.
19. Khalpey ZI, Ganim RB, Rawn JD. Postoperative care of cardiac surgery patients. In: Cohn LH, editor. Cardiac surgery in the adult. New York: McGraw-Hill; 2008. p.465-86.

20. Thompson MJ, Behramwala A, Campanella C. Immediate and long-term results of mitral prosthetic replacement using a right thoracotomy beating heart technique. Eur J Cardiothorac Surg 2003;24:47-51.

21. Katircioglu SF, Cicekcioglu F, Tutun U. On-pump beating heart mitral valve surgery without cross-clamping the aorta. J Card Surg 2008;23:307-11.

22. Cicekcioglu F, Ozen A, Tuluce H. Neurocognitive functions after beating heart mitral valve replacement without cross-clamping the aorta. J Card Surg 2008;23:114-9.

23. Karadeniz U, Erdemli O, Yamak B et . On-pump beating heart versus hypothermic arrested heart valve replacement surgery. J Card Surg 2008;23:107-13.

24. Ricci M, Macedo Fl, Suarez MRet al. Multiple valve surgery with beating heart technique. Ann Thorac Surg 2009;87:527-31.

\section{5 \\ you need not have to explain... If you lose, you should not be there to explain!}

